\title{
ANXIETY AND POST-TRAUMATIC STRESS DISORDER SYMPTOMS IN PREGNANT WOMEN DURING THE COVID-19 PANDEMIC'S DELAY PHASE
}

\author{
Meryem Hocaoglu ${ }^{1}$, Reyhan Ayaz ${ }^{1,2}$, Taner Gunay ${ }^{1}$, Elis Akin ${ }^{1}$, Abdulkadir Turgut ${ }^{1,2}$ \& Ates Karateke ${ }^{1,2}$ \\ ${ }^{l}$ Department of Obstetrics and Gynecology, Goztepe Training and Research Hospital, Istanbul Medeniyet University, \\ Istanbul, Turkey \\ ${ }^{2}$ Department of Obstetrics and Gynecology, Faculty of Medicine, Istanbul Medeniyet University, Istanbul, Turkey
}

received: 12.10.2020;

revised: 23.11.2020;

accepted: 2.12 .2020

\section{SUMMARY}

Background: The Coronavirus disease 2019 (COVID-19) pandemic emerged in Wuhan, China and has spread all over the world and affected global mental health. Pregnant women may be particularly vulnerable and experience high levels of distress during an infectious disease outbreak. The aim of this study was to determine anxiety and post-traumatic stress disorder (PTSD) symptoms in pregnant women during the COVID-19 pandemic.

Subjects and methods:This cross-sectional study surveyed a total of 283 pregnant women within the period of May 11 to May 28,2020. During their regular antenatal visit, pregnant women were invited to participate in the study. The self-created personal information form was used to assess the main characteristics of the participants. Anxiety and PTSD symptoms of the pregnant women were measured by the Spielberger State-Trait Anxiety Inventory (STAI) and Impact of Events Scale-Revised (IES-R), respectively.

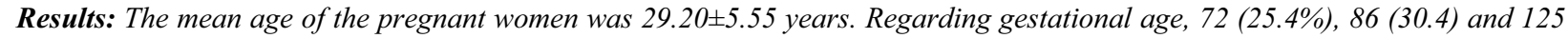
(44.2) were in the first, second and third trimesters, respectively. The mean gestational age was $23.82 \pm 11.05$ weeks. The mean STAI$S$ and STAI-T scores were $39.52 \pm 10.56$ within the cut-off value (39-40) of the instrument and 42.74 \pm 8.33 , respectively. Furthermore, the mean total IES-R score was 36.60 \pm 15.65 within the cut-off value (24) of the instrument. Multiple regression analysis revealed that pregnancy complication $(p=0.01)$ and employment status of husband $(p=0.04)$ were the best predictors of state anxiety. Additionally, the presence of COVID-19-related symptoms $(p=0.01)$ and educational level $(p=0.01)$ were found to predict PTSD symptoms.

Conclusions: Pregnant women would be likely to experience high levels of anxiety and PTSD symptoms during the COVID-19 pandemic's delay phase. The results should sensitize the medical team to increased anxiety and PTDS symptoms of the pregnant women in order to prevent negative outcomes for women and their fetuses.

Key words: Coronavirus - COVID-19 - pregnancy - anxiety - posttraumatic Stress Disorder (PTSD)

$$
* * * * *
$$

\section{INTRODUCTION}

A pneumonia of unknown cause was identified in Wuhan, China and was first reported to the WHO Country Office in China on December 31, 2019. On February 11, 2020, WHO announced a name for the new coronavirus disease: COVID-19 (Ryan et al. 2020). At the same time when governments and health agencies were trying to identify modes of transmission, strategies for disease containment and treatment for COVID-19, general fear of dying or getting in contact with individuals who are possibly infected is spreading throughout the global community and has already been reported (Šljivo et al. 2020). Besides its immediate impact on patients them- selves and on the healthcare system, this pandemic has the potential to adversely affect the mental health of millions of people (Rajkumar 2020). Taking precautions such as social isolation due to the outbreak has psychologically affected many people (Karaşar \& Canli 2020).

Pregnant women may experience particularly high levels of distress during an infectious disease outbreak for several reasons (Brooks et al. 2020). Importantly, the prenatal anxiety, depression, and stress can result in adverse birth outcomes such as miscarriage, preterm birth, low birth weight, and fetal death (Mirzadeh \& Khedmat 2020). Additionally, considerable evidence showed that if the mother is depressed, anxious, or stressed while she is pregnant her child is more likely to experience a range of adverse neurodevelopmental outcomes such as increased risk of emotional, behavioral and cognitive problems than do the children of other mothers (Glover 2014). It is important to understand psychological effects of this pandemic on pregnant women. Thus, early identification of mental health problems can allow to establish appropriate comprehensive treatment plans (Maher 2019). To date, there is limited data regarding the impact of the COVID-19 pandemic on maternal mental health (Wu et al. 2020, Saccone et al. 2020, Corbett et al. 2020).

The objective of this study was to determine anxiety and post-traumatic stress disorder (PTSD) symptoms in pregnant women during the COVID-19 pandemic's delay phase. 


\section{SUBJECTS AND METHODS}

The research protocol used in the present study was approved by the Istanbul Medeniyet University, Goztepe Training and Research Hospital, Clinical Studies Ethic Committee. Informed consent was taken from the participants. This cross-sectional study aimed to evaluate anxiety and post-traumatic stress disorder symptoms among pregnant women during the COVID-19 outbreak. From May 11 to May 28, 2020, we recruited pregnant women with singleton pregnancies from antenatal clinic of a tertiary-level Goztepe Training and Research Hospital affiliated to Istanbul Medeniyet University in Istanbul, Turkey. During their regular antenatal visit, pregnant women were invited to participate in the study, irrespective of the gestational age. Inclusion criteria were, living in Turkey, having sufficient reading and writing skills to complete self-report instruments, spoke and understood Turkish, had no loss of vision and hearing, were open to communication and cooperation, and having a confirmed pregnancy at the time of assessment. Patients were excluded if they have history of any psychiatric illness.

Data were collected using the personal information form, the Spielberger State-Trait Anxiety Inventory (STAI), and Impact of Events Scale-Revised (IES-R). The personal information form was created by members of the research team include sociodemographic data such as maternal age, gravidity, parity, smoking status, employment status, educational level, marital status, data on COVID-19 such as the presence of COVID-19related symptoms in the previous month (fever, cough, diarrhea, shortness of breath, sputum, myalgia), recent testing for COVID-19 in the past 14 days and the results of them, treatment of COVID-19, obstetric data such as pregnancy complications (threatened abortion, hyperemesis gravidarum, gestational diabetes mellitus, hypertensive disorder, placental previa, intrahepatic cholestasis of pregnancy, oligohydramnios and intrauterine growth restriction, etc.),suspected or confirmed fetal anomalies, and chronic disease (chronic hypertension, pre-gestational diabetes, cardiovascular, autoimmune, renal and hepatic diseases, etc.). The validated Turkish version of STAI was used to assess anxiety symptoms of the study participants. STAI is a Likert-type scale with two parts measuring the anxiety at a point in time (state STAI or STAI-S) and overall anxiety (trait STAI or STAI-T). Responses to the questions stating severity of the feelings, thoughts and behaviors associated with state anxiety include 'not at all, some- what, moderately so, or very much so'. Whereas the responses to the items related to trait anxiety include 'almost never, some- times, often, and almost always'. There are 20 statements in each part, and the scores range between 20 and 80 , with higher scores indicating higher anxiety levels. A cut point of $39-40$ has been suggested to detect clinically significant symptoms for the S-Anxiety scale (Julian 2011). The internal reliability for Turkish version of the Spielberger STAI was reported as $0.94-0.96$ for STAI-S and 0.83-0.87 for STAI-T. The scale also demonstrated good internal consistency and reliability in our sample (Cronbach's alpha 0.92 for STAI-S and 0.83 for STAI-T) (Öner N \& Le Compte 1983, pielberger et al 1970). IES-R measures PTSD symptoms in survivorship after an event (Hosey et al. 2019). Turkish version of IES-R was used to assess pregnant women' symptoms of post- traumatic stress in response to the COVID-19 pandemic as a potentially traumatic event (Çorapçıoğlu et al. 2006). The IES-R is a 22-item self-administered questionnaire composed of three subscales aimed to measure the mean avoidance, intrusion, and hyperarousal. Items are rated on a 5-point Likert scale, $0=$ not at all, $5=$ extremely, where scores can range from 0-88. Adaptation of IES-R to the Turkish language, achieved by Çorapcioglu et al. (2006) on 104 individuals with and 65 individuals without PTSD diagnosis, had an internal consistency coefficient of 0.94 . Also, the range for scale specifity was 70.7$81.0 \%$ and the sensitivity varied as $74.0-92.2 \%$ with respect to cut off points of 24-33 (Çorapçığlu et al. 2006, Cankardaş S \& Sofuoğlu 2019). The total IES-R score was graded for severity from normal $(0-23)$, mild (24-32), moderate (33-36), and severe psychological impact (>37) (Creamer et al 2003). A cut-off score of 24 was used to define PTSD of a clinical concern (Chew et al. 2020). Personal information form was obtained by the researcher (EA) in face-to-face interviews while waiting for their prenatal care appointment. The participants were asked to complete the IES-R and STAI. The clinical characteristics of the participants were collected by electronic medical records.

All statistical analyses were performed using SPSS 18.0. A p value of $<0.05$ was considered statistically significant. A power analysis was performed for each of the scale scores related to anxiety and PTSD symptoms by using the Univariate General Linear Model. The power of the present study was calculated as $98.9 \%$. Categorical variables were described through absolute frequencies, and continuous variables through mean and standard deviation (SD). The multiple regressions were performed with the main characteristics of the participants as predictors and their factor score on each of the state anxiety, trait anxiety and PTSD symptoms.

\section{RESULTS}

A total of 283 pregnant women were included in the study. The mean age of the pregnant women was $29.20 \pm 5.55$ years. Regarding gestational age, 72 (25.4\%), 86 (30.4) and 125 (44.2) were in the first, second and third trimesters, respectively. The mean gestational age was $23.82 \pm 11.05$ weeks. None of the enrolled pregnant women had laboratory-confirmed COVID-19, history of hospital admissions, and treatment for COVID-19. The main sociodemographic and clinical characteristics of the sample are presented in in Table 1. 
All participants completed questionnaires without data leakage. The mean state anxiety of the participating patients was found as $39.52 \pm 10.56$ within the cut-off value (39-40) of the instrument. The mean STAI-T score was $42.74 \pm 8.33$. Furthermore, the mean total IES-R score was $36.60 \pm 15.65 .215(75.9 \%)$ of the participants had an IES-R score of $\geq 24$. Out of the 283 pregnant women, $58(20.5 \%)$ women had an IES-R score of 24 32 (mild psychological impact), 25 (8.8\%) women had an IES-R score of 33-36 (moderate psychological impact), and 132 (46.6\%) women had an IES-R score of $>37$ (severe psychological impact). The scores on the administered scales are shown in Table 2.
To assess which variables were associated with anxiety and PTSD symptoms multiple regression analyses were performed. Thus, maternal age, gestational age, gravidity, parity, history of chronic disease, pregnancy complications, suspected or confirmed fetal anomalies, the presence of COVID-19-related symptoms, educational level, employment status of women, employment status of husband were evaluated in this analysis. As a result, variables of pregnancy complication $(\mathrm{p}=0.01)$ and employment status of husband $(\mathrm{p}=0.04)$ were the best predictors of state anxiety (Table 3 ). Additionally, the presence of COVID-19-related symptoms $(\mathrm{p}=0.01)$ and educational level $(\mathrm{p}=0.01)$ were found to predict PTSD symptoms.

Table 1. Sociodemographic and clinical characteristics of the participants $(n=283)$

\begin{tabular}{|c|c|c|c|}
\hline Characteristics & $\mathrm{n}(\%)$ & Mean & Standard deviation \\
\hline Maternal age (years) & & 29.20 & 5.55 \\
\hline Gestational age (weeks) & & 23.82 & 11.05 \\
\hline Gravidity & & 2.48 & 1.48 \\
\hline Parity & & 1.06 & 1.03 \\
\hline \multicolumn{4}{|l|}{ Trimester } \\
\hline First trimester & $72(25.4)$ & & \\
\hline Second trimester & $86(30.4)$ & & \\
\hline Third trimester & $125(44.2)$ & & \\
\hline Current smoker (yes) & $26(9.3)$ & & \\
\hline Marital status (Married) & $273(96.5)$ & & \\
\hline Employment status of pregnant women (Employed) & $62(21.9)$ & & \\
\hline Employment status of husbands ${ }^{\S}$ (Employed) & $252(89.0)$ & & \\
\hline \multicolumn{4}{|l|}{ Educational level } \\
\hline Illiterate & $1(0.3)$ & & \\
\hline Primary school & $58(20.5)$ & & \\
\hline Secondary school & $60(21.2)$ & & \\
\hline High school & $91(32.2)$ & & \\
\hline University & $73(25.8)$ & & \\
\hline Pregnancy complications (Yes) & $54(19.1)$ & & \\
\hline Suspected or confirmed fetal anomalies (Yes) & $12(4.3)$ & & \\
\hline History of chronic disease (Yes) & $65(23.0)$ & & \\
\hline Presence of COVID-19-related symptoms in the previous month* (Symptomatic) & $86(30.4)$ & & \\
\hline Recent testing for COVID-19 in the past 14 days ${ }^{*}($ Yes $)$ & $9(3.2)$ & & \\
\hline
\end{tabular}

Table 2. Anxiety and post-traumatic stress disorder symptoms of the participants

\begin{tabular}{lc}
\hline Measures & $\mathrm{N}=283$ Mean \pm SD \\
\hline STAI (state) & $39.52 \pm 10.56$ \\
STAI (trait) & $42.74 \pm 8.33$ \\
IES-R (total) & $36.60 \pm 15.65$ \\
IES-R 0-23 (normal) & $68(24 \%)$ \\
IES-R 24-32 (mild psychological impact) & $58(20.5 \%)$ \\
IES-R 33-36 (moderate psychological impact) & $25(8.8 \%)$ \\
IES-R >37 (severe psychological impact) & $132(46.6 \%)$ \\
\hline
\end{tabular}

Abbreviations: STAI, State-Trait Anxiety Inventory; IES-R, Impact of Event Scale-Revised

Data are presented as number (percentage) or as mean \pm standard deviation 
Table 3. Multiple regressions assessing the relationship between sociodemographic and clinical characteristics and anxiety and post-traumatic stress disorder symptoms of the participants

\begin{tabular}{|c|c|c|c|c|c|c|c|}
\hline Criteria - Predictors & $\begin{array}{l}\text { Adjus } \\
\text { ted } \mathrm{R}^{2}\end{array}$ & B & SE & $\beta$ & p-value & $\begin{array}{c}95 \% \mathrm{CI} \\
\text { lower bound }\end{array}$ & $\begin{array}{c}95 \% \mathrm{CI} \\
\text { upper bound }\end{array}$ \\
\hline State anxiety & 0.036 & & & & & & \\
\hline Maternal age & & 0.05 & 0.13 & 0.02 & 0.71 & -0.21 & 0.30 \\
\hline Gestational age & & -0.03 & 0.06 & -0.03 & 0.61 & -0.15 & 0.09 \\
\hline Gravidity & & -0.13 & 0.74 & -0.02 & 0.87 & -1.58 & 1.33 \\
\hline Parity & & 1.36 & 1.09 & 0.13 & 0.21 & -0.78 & 3.51 \\
\hline History of chronic disease & & 1.32 & 1.52 & 0.05 & 0.38 & -1.67 & 4.31 \\
\hline Pregnancy complications & & 4.24 & 1.64 & 0.16 & $0.01 *$ & 1.00 & 7.48 \\
\hline Suspected or confirmed fetal anomalies & & 0.68 & 3.10 & 0.01 & 0.83 & -5.43 & 6.79 \\
\hline Presence of COVID-19-related symptoms & & 0.94 & 1.37 & 0.04 & 0.49 & -1.75 & 3.63 \\
\hline Educational level & & -0.73 & 0.66 & -0.08 & 0.27 & -2.03 & 0.57 \\
\hline Employment status of pregnant women & & 0.66 & 1.58 & 0.03 & 0.68 & -2.45 & 3.76 \\
\hline Employment status of husbands ${ }^{\S}$ & & -4.19 & 2.03 & -0.12 & $0.04 *$ & -8.19 & -0.18 \\
\hline Trait anxiety & 0.018 & & & & & & \\
\hline Maternal age & & 0.10 & 0.10 & 0.07 & 0.33 & -0.10 & 0.30 \\
\hline Gestational age & & 0.01 & 0.05 & 0.01 & 0.83 & -0.08 & 0.10 \\
\hline Gravidity & & -0.40 & 0.59 & -0.07 & 0.50 & -1.56 & 0.77 \\
\hline Parity & & 0.80 & 0.87 & 0.10 & 0.36 & -0.92 & 2.52 \\
\hline History of chronic disease & & 0.70 & 1.21 & 0.04 & 0.57 & -1.69 & 3.09 \\
\hline Pregnancy complications & & 1.52 & 1.32 & 0.07 & 0.25 & -1.07 & 4.11 \\
\hline Suspected or confirmed fetal anomalies & & -2.13 & 2.48 & -0.05 & 0.39 & -7.01 & 2.76 \\
\hline Presence of COVID-19-related symptoms ${ }^{\$}$ & & 1.52 & 1.09 & 0.08 & 0.17 & -0.63 & 3.67 \\
\hline Educational level & & -0.87 & 0.53 & -0.11 & 0.10 & -1.91 & 0.18 \\
\hline Employment status of pregnant women & & -1.15 & 1.26 & -0.06 & 0.36 & -3.64 & 1.33 \\
\hline Employment status of husbands ${ }^{\S}$ & & -0.88 & 1.63 & -0.03 & 0.59 & -4.08 & 2.32 \\
\hline PTSD symptoms & 0.068 & & & & & & \\
\hline Maternal age & & 0.02 & 0.19 & 0.01 & 0.93 & -0.35 & 0.38 \\
\hline Gestational age & & -0.07 & 0.09 & -0.05 & 0.41 & -0.24 & 0.10 \\
\hline Gravidity & & -0.94 & 1.07 & -0.09 & 0.38 & -3.05 & 1.17 \\
\hline Parity & & 2.12 & 1.58 & 0.14 & 0.18 & -0.99 & 5.22 \\
\hline History of chronic disease & & 3.24 & 2.20 & 0.09 & 0.14 & -1.08 & 7.57 \\
\hline Pregnancy complications & & 3.85 & 2.38 & 0.10 & 0.11 & -0.84 & 8.53 \\
\hline Suspected or confirmed fetal anomalies & & 0.60 & 4.49 & 0.01 & 0.89 & -8.24 & 9.44 \\
\hline Presence of COVID-19-related symptoms ${ }^{\$}$ & & 4.90 & 1.98 & 0.15 & $0.01 *$ & 1.00 & 8.79 \\
\hline Educational level & & -2.50 & 0.96 & -0.17 & $0.01 *$ & -4.38 & -0.61 \\
\hline Employment status of pregnant women & & -3.05 & 2.28 & -0.08 & 0.18 & -7.55 & 1.45 \\
\hline Employment status of husbands ${ }^{\S}$ & & -0.64 & 2.94 & -0.01 & 0.83 & -6.43 & 5.15 \\
\hline
\end{tabular}

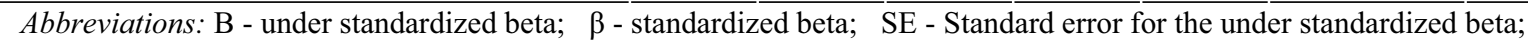

$\mathrm{P}$ - probability value; CI - confidence intervals; PTSD - post-traumatic stress disorder

Included threatened abortion, hyperemesis gravidarum, gestational diabetes mellitus, hypertensive disorder, placental previa, intrahepatic cholestasis of pregnancy, oligohydramnios and intrauterine growth restriction, and high-risk pregnancy status.

${ }^{\sharp}$ Included fever, cough, diarrhea, shortness of breath, sputum, and myalgia. ${ }^{\S}$ If is married.

Three multiple regressions were performed with the main clinical and sociodemographic characteristics of the participants as predictors and their factor score on each of the state anxiety, trait anxiety and PTSD symptoms.

${ }^{*} \mathrm{p}<0.05$ was considered statistically significant.

\section{DISCUSSION}

This cross-sectional survey study investigated anxiety and PTSD symptoms in pregnant women during the COVID-19 pandemic's delay phase. Our results revealed that the pandemic has a negative emotional impact on pregnant women, creating anxiety, and PTSD symptoms. Moreover, while pregnancy complication and employment status of husband were found to be the best predictors of state anxiety, the presence of COVID-19related symptoms and educational level were found to predict PTSD symptoms.

Studies with measures of mental health reported high levels of anxiety in pregnant women during infec- tious disease outbreaks (Brooks et al. 2020). It may be expected result in early phase of disease. However, the prolonged pandemic chaos will inevitably have economic consequences, and financial uncertainties are likely to further escalate psychological burden and worsen the mental wellbeing of pregnant women (Karaşar \& Canli 2020). The first case of COVID-19 in Turkey was detected on 11 March 2020, and the first death was reported on 17 March. After the first case was detected, radical interventions were implemented by the Turkish government to prevent the spread of the disease (Durankuş \& Aksu 2020). The present study was conducted in a period which represented the COVID-19 pandemic's delay phase. In fact, the radical interventions were 
continued in the study period. Nevertheless, despite reassuring findings including increased available information and reassurance through social media, healthcare professionals and primary care (Kotabagi et al. 2020), our study showed that the pregnant women reported high anxiety levels during the COVID-19 pandemic's delay phase. The mean STAI-S scores were above the cut-off that detect clinically significant symptoms for the state anxiety. The state anxiety reflects the pregnant women' anxiety at the time the COVID pandemic. The pregnant women also reported higher levels of trait anxiety. As trait anxiety reveals the overall anxiety of a person and is related to personality type. Unlike our results, Kotabagi et al. (2020) examined the anxiety and depression levels among pregnant women with COVID-19 at the tail-end of the pandemic in the UK and suggested that maternal levels of anxiety appear low, with depression levels following a similar pattern. However, that study is a pilot study which enrolled a small number of participants. Nevertheless, most previous studies investigated stress and the anxiety levels of pregnant women in early phase of the disease (Wu et al. 2020, Saccone et al. 2020, Corbett et al. 2020, Lebel et al. 2020, Berthelot et al. 2020).

In consistent with our results, $\mathrm{Wu}$ et al. indicated that there is a clinically significant increase in the prevalence of depressive and anxiety symptoms after the declaration of human-to-human transmission and an increased threat of the COVID-19 epidemic (Wu et al. 2020). In a study by Lebel et al. (1987) pregnant participants were surveyed across Canada in April 2020 and depression, general anxiety, and pregnancy-specific anxiety symptoms were all substantially higher than in previous community pregnancy cohorts with similar demographic profiles during the pandemic (Lebel et al. 2020).

Not surprising, we found that the mean IES-R scores were above the cut-off score for PTSD symptoms. $46.6 \%$ of participants had an IES-R score of $>37$ which represents severe psychological impact. Similar to our research, Saccone et al. (2020) conducted a survey study in Napoli, Italy from March 15, 2020 to April 1, 2020 and reported that the COVID-19 pandemic had a moderate psychological impact on pregnant women with a mean IES-R score of $36.9 \pm 10.1$. They also remarked that mean score at STAI questionnaire was 45.2 \pm 14.6 . Indeed, that study had small sample size and the main characteristics of the participants were not clearly reported. Furthermore, Berthelot et al. (2020) evaluated two cohorts of pregnant women, one that was recruited before the COVID-19 pandemic in Quebec, Canada; the other $(n=1258)$ was recruited online during the pandemic from April 2 to April 13 2020. Similar to our findings, they suggested that pregnant women assessed during the COVID-19 pandemic reported higher levels of depressive and anxiety symptoms, dissociative symptoms, and symptoms of post-traumatic stress disorder, negative affectivity and less positive affectivity than the pre-COVID-19 cohort.

Notably, we found that pregnancy complication and employment status of husband were the best predictors of state anxiety. These results indicated that financial and occupational concerns and related health risks to both the self and unborn babies may create creating a negative impact on pregnant women (Brooks et al. 2020). Moreover, the presence of COVID-19-related symptoms and educational level were found to predict PTSD symptoms. It was known that high educational level was among the factors that increased the risk of PTSD (Bromet et al 2017).

COVID-19 presents serious psychological challenges for pregnant individuals, with the potential for both short term and long-lasting impacts on the developing fetus (Lebel et al. 2020). In this context, our research underscores the need for further research on the effects the long-term consequence of stress and anxiety and their impact on perinatal outcomes and fetal neurobehavioral development. It should be noted that, anxiety, as a kind of psychological stress, will trigger a series of physiological events and cause a decrease in immunity (HasanpourDehkordi et al. 2019). Thus, the interventions for perinatal mental health should be a priority during any public-wide epidemic to reduce psychological distress during pregnancy (Mirzadeh \& Khedmat 2020).

This study has notable strengths. Our study has an adequate sample size. Survey data was collected through a face-to-face interview by the same researcher. Another strength of the present study includes detailed data for sociodemographic and clinical characteristics of the participants. There are, however, limitations of our research. First limitation is due to the self-reporting of the levels of psychological impact, anxiety, depression and stress, which may not always be aligned with objective assessment by mental health professionals (Wang et al. 2020). However, it could not be possible during the COVID-19 crisis. Secondly, our results were limited by the single center study design.

\section{CONCLUSIONS}

The findings of our study indicated that pregnant women would be likely to experience high levels of anxiety and PTSD symptoms during the COVID-19 pandemic's delay phase. The results should sensitize the medical team to increased anxiety and PTDS symptoms of the pregnant women in order to prevent negative outcomes for women and their fetuses.

\section{Contribution of individual authors:}

Meryem Hocaoglu: idea, concept and design of the article, interpretation of data, first manuscript draft.

Reyhan Ayaz: concept and design of the article, first manuscript draft.

Taner Gunay: data collection, literature review, statistical analysis.

Elis Akin: data collection, literature review.

Abdulkadir Turgut \& Ates Karateke:: supervision.

All authors approval of the final version. 
Acknowledgements: None.

\section{Conflict of interest: None to declare.}

\section{References}

1. Berthelot N, Lemieux R, Garon-Bissonnette J, DrouinMaziade C, Martel É, Maziade M: Uptrend in distress and psychiatric symptomatology in pregnant women during the COVID-19 pandemic. Acta Obstet Gynecol Scan 2020. https://doi.org/10.1111/aogs.13925

2. Bromet EJ, Atwoli L, Kawakami N, Mateu FN, Piotrowski $P$, King AJ et al: Post-traumatic stres disorder associated with natural and human-made disasters in the World Mental Health Surveys. Psychological Medicine 2017; 47:227-41

3. Brooks S, Weston D, Greenberg N: Psychological impact of infectious disease outbreaks on pregnant women: Rapid evidence review. medRxiv preprint 2020. https://doi.org/10.1101/2020.04.16.20068031

4. Cankardaş S \& Sofuoğlu Z: Post-Traumatic Stress Disorder Symptoms and Their Predictors in Earthquake or Fire Survivors. Turk Psikiyatri Derg 2019; 30:151-156

5. Chew NW, Lee GK, Tan BY et al: A multinational, multicentre study on the psychological outcomes and associated physical symptoms amongst healthcare workers during COVID-19 outbreak. Brain Behav Immun 2020; 88:559-565

6. Corapcjog Чu A, Yargıc, I, Geyran P et al: Validity and Reliability of Turkish Version of "Impact of Event ScaleRevised" (IES-R). New Symposium Journal 2006; 44:14-22

7. Corbett GA, Milne SJ, Hehir MP, Lindow SW, O'Connell M: Health anxiety and behavioural changes of pregnant women during the COVID-19 pandemic. Eur J Obstet Gynecol Reprod Biol 2020; 249:96-97

8. Creamer M, Bell R, Failla S: Psychometric properties of the Impact of Event Scale Revised. Behav Res Ther 2003; 41:1489-1496

9. Durankuş $F$ \& Aksu E: Effects of the COVID-19 pandemic on anxiety and depressive symptoms in pregnant women: a preliminary study. J Matern Fetal Neonatal Med 2020. https://doi.org/10.1080/14767058.2020.1763946

10. Glover V: Maternal depression, anxiety and stress during pregnancy and child outcome; what needs to be done. Best Pract Res Clin Obstet Gynaecol 2014; 28:25-35

11. Hasanpour-Dehkordi A, Solati K, Tali SS, Dayani MA: Effect of progressive muscle relaxation with analgesic on anxiety status and pain in surgical patients. $\mathrm{Br} J$ Nurs 2019; 28:174-178

12. Hosey M, Bienvenu OJ, Dinglas VD, Turnbull AE, Parker $A M$, Hopkins RO et al: The IES-R remains a core outcome measure for PTSD in critical illness survivorship research. Crit Care 2019; 23:362

13. Julian LJ: Measures of anxiety: State-Trait Anxiety Inventory (STAI), Beck Anxiety Inventory (BAI), and Hospital Anxiety and Depression Scale-Anxiety (HADSA). Arthritis Care Res (Hoboken) 2011; 63(S11):S467S472

14. Karaşar B \& Canli D: Psychological Resilience and Depression during the COVID-19 Pandemic in Turkey. Psychiatr Danub 2020; 32:273-279

15. Kotabagi P, Fortune L, Essien S, Nauta M, Yoong W: Anxiety and depression levels among pregnant women with COVID-19 Acta Obstet Gynecol Scand 2020; 99:953-954

16. Lebel C, MacKinnon A, Bagshawe M, Tomfohr-Madsen L, Giesbrecht G: Elevated depression and anxiety among pregnant individuals during the COVID-19 pandemic. J Affect Disord 2020. https://doi.org/10.1016/j.jad.2020.07.126

17. Maher M: Emergency Preparedness in Obstetrics: Meeting Unexpected Key Challenges. J Perinat Neonatal Nurs 2019; 33:238-245

18. Mirzadeh $M$ \& Khedmat L: Pregnant women in the exposure to COVID-19 infection outbreak: the unseen risk factors and preventive healthcare patterns. J Matern Fetal Neonatal Med 2020. https://doi.org/10.1080/14767058.2020.1749257

19. Ö̈er $N \&$ Le Compte A: Durumluk-sürekli kaygl envanteri el kitabı. Istanbul, TU: Bogăzicj Uñiversitesi yayınları; 1983 (in Turkish)

20. Rajkumar RP: Attachment Theory and Psychological Responses to the COVID-19 Pandemic: A Narrative Review. Psychiatr Danub 2020; 32:256-261

21. Ryan GA, Purandare NC, McAuliffe FM, Hod M, Purandare CN: Clinical update on COVID-19 in pregnancy: A review article. J Obstet Gynaecol Res 2020; 46:1235-1245

22. Saccone G, Florio A, Aiello F et al: Psychological Impact of COVID-19 in pregnant women. Am J Obstet Gynecol 2020; 223:293-295

23. Šljivo A, Kačamaković M, Quraishi I, Džubur Kulenović A: Fear and Depression among Residents of Bosnia and Herzegovina during COVID-19 Outbreak - Internet Survey. Psychiatr Danub 2020; 32:266-272

24. Spielberger CD, Gorsuch RL, Lushene RE: Manual for State-Trait Anxiety Inventory. California: Consulting Psychologist Press, 1970

25. Wang $C$, Pan R, Wan X et al: A longitudinal study on the mental health of general population during the COVID-19 epidemic in China. Brain Behav Immun 2020; 87:40-48

26. Wu Y, Zhang C, Liu H, Duan C, Li C, Fan J et al: Perinatal depressive and anxiety symptoms of pregnant women along with COVID-19 outbreak in China. Am J Obstet Gynecol 2020; 223:240.e1-240.e9

Correspondence:

Meryem Hocaoğlu, MD

Department of Obstetrics and Gynecology, Goztepe Training and Research Hospital

Kadıkoy, Istanbul, Turkey

E-mail:dr.meryemtaskiran@gmail.com 